\title{
La espacialidad cultural del riesgo: construcción de enfoques para su análisis y reflexión
}

\author{
The cultural spatiality of risk: construction of approaches for analysis and reflection \\ Adriana Lucia Trejo Albuerne ${ }^{a}$, Bonnie Lucía Campos Cámara ${ }^{b}$
}

\begin{abstract}
:
This essay addresses a reflection that arises from the concern to know the relationship between space and culture to the phenomena associated with the different risk scenarios. Considering an approximation from an integral vision through different types of spaces, the cultural spatiality of risk will be called where an emphasis is placed on the sociocultural construction of risk. A proposal will be made from a multidisciplinary approach that requires reading from a community, that it contains multiple visions and perceptions of the same phenomenon: risk. With the above, it is intended to open a debate or initial reflections on the incidence and relevance that it would have in its application in a given space and experiment in other territorial proposals that help mitigate pre-existing risks and not generate new ones in the territories.
\end{abstract}

\section{Keywords:}

Geography, Cultural spatiality, Risk, Proposal

\section{Resumen:}

Este ensayo aborda una reflexión que surge de la inquietud por conocer la relación entre el espacio y la cultura ante los fenómenos asociados con los diferentes escenarios de riesgo. Considerando una aproximación desde una visión integral a través de diferentes tipos de espacios, se denominará la espacialidad cultural del riesgo, donde se hace énfasis en la construcción sociocultural del riesgo. Se hará una propuesta desde un enfoque multidisciplinario que requiere una lectura desde una colectividad, que a su vez, encierra múltiples visiones y percepciones sobre el mismo fenómeno: el riesgo. Con lo anterior, se pretende abrir un debate o reflexiones iniciales sobre la incidencia y relevancia que tendría la espacialidad cultural del riesgo en su aplicación en un espacio determinado y experimentar en otras propuestas territoriales que ayuden a mitigar los riesgos preexistentes y no generar nuevos en los territorios.

\section{Palabras Clave:}

Geografía, espacialidad cultural, riesgo, propuesta

Ensayo recibido: 12 de septiembre de 2019

Dictaminado: 16 de octubre de 2019

Segunda versión: 21 de octubre de 2019

Aceptado: 25 de octubre de 2019

\footnotetext{
a Autor de Correspondencia, estudiante del Doctorado en Geografía en la Universidad de Quintana Roo, Email: albuerneadriana@yahoo.com

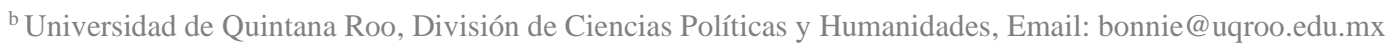




\section{Introducción}

Esta reflexión parte desde el marco donde los riesgos se entienden como construcciones humanas y culturales que dependen de factores culturales vinculados a estructuras sociales. Este tipo de enfoque ha sido llevado por disciplinas como la antropología cultural. Con esto, se entiende que el riesgo se contempla como algo que puede cambiar a través de la intervención humana.

La temática se abordará, en en la parte inicial, con algunas aproximaciones al riesgo y a la cultura desde la Geografía; se hará énfasis en la visión desde la Geografía, para después hacer un análisis de la relación entre cultura y el riesgo. Con ello se da pie a una propuesta inicial de análisis de los espacios sociales y territoriales en situación de riesgo, a través de una espacialidad integral que queda abierta para sumarse a otros análisis o aplicaciones territoriales en proyectos futuros en estudios de caso definidos.

\section{Aproximaciones al riesgo y la cultura desde la Geografía}

Tradicionalmente, se proporciona información valiosa sobre procesos espaciales y sociales específicos relacionados con diversos riesgos. Sin embargo, son necesarias nuevas conceptualizaciones integradoras que incluyan ideas de diversas corrientes, ciencias naturales, humanidades y ciencias sociales. Es así como el objeto de estudio de la Geografía, por su misma naturaleza de carácter integrador y visión holística, la coloca como una de las disciplinas más pertinentes para las necesidades actuales ante los estudios y acercamientos teóricos y prácticos de los riesgos.

El riesgo es un peligro que se ha calculado a través de un conjunto completo de percepciones, negociaciones y acciones, la construcción social del riesgo significa convertir las contingencias en algo accesible, evaluable, mensurable y manejable (Kruger 2013). Sin importar cuál sea el enfoque desde el que se mire, siempre, desde sus primeras concepciones, aparece ligado a la inseguridad, a la falta de capacidad de conocer el futuro y a la posibilidad de sufrir daños (Toscana, 2006).

El concepto de riesgo a lo largo de su existencia, ha sido de gran amplitud. El término ha sido retimado por varias disciplinas, como la Economía, la Medicina (Rojas y Martínez, 2011), la Sociología, la Antropología, la Psicología, la Ingeniería, la Geografía y casi todos los campos disciplinarios de las Humanidades, las Ciencias y la Tecnología (Toscana, 2006), por lo que buscar un solo significado es complicado, debido a que cada disciplina le atribuye su propia valoración (Cardona, 2001).

Mientras Toscana Aparicio (2006) menciona que el concepto de riesgo podría venir del árabe clásico rizq, cuyo significado es lo que depara la Providencia, Aneas de Castro (2000) confirma como incierto su origen, al afirmar que procede del latín resecare: cortar; etimológicamente, riesgo proviene de rísico o rischio (peligro) entre otros significados.

Hewitt (1997) describe cuatro factores que determinan los riesgos: los eventos peligrosos, la vulnerabilidad social y la adaptación a las amenazas; los rasgos estructurales de los entornos naturales y sociales (hábitat) $y$, las estrategias de supervivencia y las medidas de intervención. El riesgo se convierte así en una característica no sólo de la cultura y la sociedad, sino también de una región específica de la localización (Krüger et al., 2015).

El estudio de riesgos y desastres no se reconoció como tema científico hasta mediados del siglo $\mathrm{XX}$, pero este reconocimiento se sustentó en una tradición antigua, en textos geográficos, históricos y de otras temáticas, lo que sugiere que la teoría de riesgos de desastres es producto de las experiencias de las sociedades y de las exploraciones de los territorios. Por su parte, la palabra desastre que proviene del prefijo latino des (falta de, malo) y de la palabra griega astron o astren (estrella) cuyo significado literal sería mala estrella, al relacionar fenómenos de origen natural peligrosos (Martínez, 2011). Es importante considerar que en los estudios del desastre se involucran múltiples factores como: la relación entre peligro, desastres, amenazas y conocimiento local; las interpretaciones, valores y tabúes; las experiencias, prioridades y acciones de la gente vulnerable; las relaciones de poder en el estudio de cómo se construye el riesgo; la integración de los riesgos y desastres en el contexto de justicia y derechos humanos; la naturaleza de las estrategias globalizadoras de manejo del riesgo, el desajuste de las tradiciones y las tecnologías y políticas modernas de la Reducción del Riesgo del Desastre (RRD) (Krüger et al., 2015).

Fred Krüger (2015) menciona que a lo largo de las últimas décadas, se ha manifestado una inversión académica sustancial en el papel que la cultura tiene en relación con los desastres. Sin embargo, ha crecido la tasa de pérdidas tanto humanas como materiales. Asimismo, en las dos últimas décadas del siglo pasado, se han masificado los estudios sobre desastres, y en la actualidad, estos análisis no escapan a ningún ámbito de acción humana en las sociedades contemporáneas.

Algunos ejemplos de estudios por todo el mundo y que no se concentran únicamente en amenazas o peligros de origen natural, han incluido temas de epidemias y salud, como estudios de caso sobre el Síndrome de Inmunodeficiencia Adquirida (conocido por sus siglas SIDA, o AIDS en inglés) $u$ otras temáticas como el terrorismo, el calentamiento global, químicos y todos los 
peligros que se derivan del uso de grandes sistemas tecnológicos, como la energía nuclear (Toscana, 2006).

La eficiencia de manejar los peligros depende de la forma en que la población los percibe; para que un grupo social trate con éxito las amenazas, debe ser capaz de articularlas y abordarlas. Las amenazas deben ser socialmente adoptadas y traducidas en riesgos (Kruger, 2013).

Alrededor del mundo y a varias escalas, se han establecido distintas instituciones de diversos orígenes (gubernamentales, privadas, civiles, etcétera) para tratar con los temas de desastres, sobre las cuales destaca la United Nations Office for Disaster Risk Reduction (UNISDR), creada en 1999. Es el punto de enlace dentro del Sistema de la ONU para promover sinergias y la coordinación entre las diversas actividades para la reducción de desastres en los campos socioeconómico, humanitario y de desarrollo, así como para brindar apoyo a la integración de las diversas políticas afines.

En 2013, como consecuencia de un furor por los temas del riesgo, Müller-Mahn y algunos otros autores publican The Spatial Dimension of Risk How geography shapes the emergence of riskscapes, obra fundamental para esta aproximación, donde se hace un acercamiento teórico y metodológico sustentado por distintos escenarios y paisajes del riesgo en diversos estudios de caso y desde distintas perspectivas constructivistas, pero desde la espacialidad: la Geografía. (Müller-Mahn, 2013). Otra recopilación destacada es Cultures and Disasters. Understanding cultural framings in disaster risk reduction, editada por Fred Krüger, Greg Bankoff, Terry Cannon, Benedikt Orlowski y Lisa Schipper a través de una compilación de los trabajos resultantes de los debates realizados en las conferencias internacionales: Cultures and Disasters, celebrada en julio de 2011 y Cultures and Disasters II: Exploring the Links between Disasters and Culture(s): Preparedness, Response, Policies, estas últimas en julio de 2013, en Alemania. En estos trabajos es posible encontrar temas y estudios de casos diversos desde la relación entre la cultura y los desastres, considerando la óptica de los principales investigadores de talla internacional, hasta enfoques interdisciplinarios (Krüger et al., 2015).

En México existen algunos precedentes de estudios cuyo objetivo es la relación que existe entre el desastre y la cultura. Alejandra Toscana Aparicio (2006 y 2014) ha trabajado, en su tesis de doctorado Los paisajes del desastre y las Representaciones sociales del desastre de 1940 en Santa Cruz Pueblo Nuevo, Estado de México, en la que propone una aproximación para el estudio del paisaje, considerándolo como un conjunto de elementos tanto físico-naturales como sociales, configurado a través de procesos histórico-culturales, susceptible de ser representados simbólicamente. Analiza tres diversas maneras de la relación del espacio físico cultural, que dan lugar a variadas formas de representación del espacio, las cuales ordenan la experiencia espacial y producen identidades.

A lo largo del tiempo, todas las sociedades han elaborado una cultura del espacio en torno de diversas prácticas espaciales, como crear y ordenar espacios productivos, establecer normas urbanísticas, modificar los paisajes, acondicionar áreas con funciones sociales específicas, delimitar y separar espacios, ejercer dominio sobre los mismos, ubicarlos, ordenarlos, describirlos y establecer relaciones más o menos precisas de los elementos que los constituyen, lo que ha producido a su vez, cierta imagen del espacio y cierto saber espacial, que tiene un carácter cultural y un valor político que se denomina Geografía (Ortega, 2000). El geógrafo considera como fundamental al individuo y a la sociedad que transforma, vive y construye el espacio y es de interés estudiar estos cambios desde el concepto de espacio físico hasta el territorio como espacio vivido y construido (Vargas, 2012).

Lo anterior representa a su vez, una orientación más pragmática de la Geografía y más cercana a la sociedad, a los problemas del mundo real y a las necesidades de lo privado y público (Buzai, López Trigal, en Garrocho et al., 2015). Puede ser una solución al acercamiento de los riesgos, ya que su capacidad para intercambiar y acomodar coherentemente el conocimiento que proviene de múltiples disciplinas y construir sinergias contemplando su espacialidad y temporalidad, se relaciona de forma lógica, convergente e innovadora para entender al entorno. Asimismo, cuenta con una construcción permanente de nuevos métodos de análisis espaciotemporal multiescalar, donde la localización, distribución, distancia, escala y tiempo son incorporados de manera explícita, tanto en la etapa analítica como en la explicativa (Garrocho et al., 2015).

En 1984, Calvo García define una Geografía de los Riesgos, en la que une los componentes físicos con componentes sociales. Afirmó que el estudio de los riesgos naturales se encuentra en los estadíos primarios del proceso de investigación, para ese momento no existía una teoría general, aunque se hayan hecho aportaciones importantes. Los estudios geográficos sobre el riesgo, muestran una capacidad clara para afrontar temas de actualidad, así como contribuir con soluciones reales (Calvo, 1984). Finalmente, es importante señalar la importancia de la injerencia geográfica por la compleja y siempre dinámica espacial, entre los riesgos, desastres, el entorno y las manifestaciones territoriales del ser humano en su incidencia y modificación espacial. 


\section{La relación entre la cultura y el riesgo}

El desafío de acercar a la cultura en la discusión del riesgo, existe no sólo porque la cultura en sí misma es un tema controvertido sino también porque supone un componente principal que se manifiesta de múltiples maneras dentro de los riesgos (Krüger et al., 2015). El área cultural, en tanto que comunidad, con una forma de vida, es un crecimiento que ocurre en un espacio particular, una expresión histórico-geográfica, donde los modos de vida y economía en general, son la representación de una adaptación ambiental (Vargas, 2012).

El riesgo es un resultado de las percepciones, decisiones $y$ acciones de la sociedad $y$, por lo tanto, una construcción social. Frente a la incertidumbre del futuro y los posibles peligros que éste pudiera traer consigo, se han construido conocimiento y técnicas que van desde la adivinación hasta la elaboración de sofisticados cálculos de probabilidades y modelos (Toscana, 2006). Desde las teorías culturales, se sostiene que las fuentes de riesgo se perciben dependiendo del sistema de creencias y valores de cada cultura, por lo que al identificar los diferentes sistemas de creencias y valores en función del riesgo ante fenómenos de origen natural, se proporcionan elementos base para definir con mayor precisión planes de reducción de riesgo con mayor probabilidad de éxito al momento de su aplicación (UNISDR, 2015).

A partir de la perspectiva cultural, los individuos y los grupos sociales poseen una percepción sesgada de la realidad, en función de sus valores culturales, sus experiencias o bien sus aspiraciones (Capel, 2010). Así, la diversidad de los puntos de vista relativos a un riesgo determinado no dependen solamente del nivel de competencia técnica de cada individuo y no se limita, por lo tanto, a una oposición entre experto (científico) y ciudadano ordinario (Krüger et al., 2015).

La comprensión de la cultura reviste una importancia significativa, puesto que denota las consideraciones que dictarán determinadas reacciones ante los riesgos y resulta pertinente para orientar la ejecución de las actividades orientadas a la reducción del riesgo de desastres. Además, la cultura puede agudizar o disminuir la vulnerabilidad. La interacción entre la cultura y el riesgo está ligada a numerosos aspectos del comportamiento, incluidos la religión, los medios de vida, la percepción del riesgo, la interacción con los demás miembros de la comunidad y la importancia de las relaciones de poder, el lugar de residencia y los efectos de la cultura en la edificación de inmuebles y la salud (UNISDR, 2015).

El riesgo, abordado como un fenómeno representacional que deriva en una construcción social, se encuentra ligado a procesos que poseen una manifestación territorial innegable — desde sus dimensiones físicas y simbólicas - y que, por tanto, influyen directamente en los factores que generan dicha construcción. El riesgo, como construcción social, se remite al entendimiento de los saberes, conocimientos, visiones y cosmovisiones vinculados a la noción de territorio.

En la íntima naturaleza humana, indisoluble de su entorno, el riesgo guarda una estrecha relación con el diálogo que se ha desarrollado históricamente entre el medio y sus habitantes; una cotidianidad que le permite al individuo en comunidad identificar los acontecimientos que pueden poner en peligro esta comunicación. Tal es el caso de los desastres, procesos que modifican temporal o permanentemente el espacio, alterando las vías de interacción. Se manifiesta y se resalta así el vínculo desastre- riesgo- territorio. Desde estos aspectos, en los siguientes párrafos, se intentará construir un concepto sobre territorialidad, teniendo algunas consideraciones que se inscriben en el ámbito cultural y geográfico.

\section{La espacialidad integral del riesgo}

El espacio ha sido, de una forma u otra, como un fondo o como expresión directa de las preocupaciones geográficas. Sin embargo, al igual que la Geografía y la ciencia en general, este concepto se ha ido modificando y se ha contemplado de distintas maneras a lo largo del tiempo (Ortega, 2000). Si bien, el modo de entender el espacio difiere, las diversas categorías de análisis espacial que se manifiestan en éste (lugares, paisaje, regiones y espacio social, entre otras), se manifiestan en distintas formas para representar el espacio como objeto geográfico.

Para Milton Santos, el espacio está conformado por un conjunto indisoluble, solidario y contradictorio de sistemas de objetos y sistemas de acciones, que no están aislados sino como parte del contexto en el que se realiza la historia. Su construcción depende de la interacción de los humanos, las empresas, las instituciones, el medio ecológico y las infraestructuras (Martínez, 2011). En este sentido, el espacio proporciona los elementos para la superposición de múltiples riesgos en lugares y regiones particulares. Éste puede ser abordado como un marco analítico para el estudio del riesgo y como una herramienta empírica para la gestión de riesgos, basada en la localización, medición, regionalización y cartografía de riesgos particulares (Müller-Mahn, 2013).

El espacio a su vez, es una propiedad de los objetos y la configuración territorial, la expresión de esa espacialidad. La espacialidad humana conlleva que el espacio forme parte inseparable de la práctica social y que, por ello, las nociones espaciales, de igual modo que las metáforas 
espaciales, constituyan un componente habitual del lenguaje (Ortega, 2000).

Peter Weichhart y Karl-Michael Höferl (en Müller-Mahn, 2013) realizan un análisis a partir de una perspectiva constructivista y pragmática de los términos espacio, riesgo y sus distintos significados a lo largo del tiempo, así como las posibles relaciones que existen entre ambos conceptos desde una visión socio-geográfica. Afirman que, en el campo de la investigación de riesgos, se debe tener en cuenta el espectro de significado de estos conceptos y su contexto, lo que resulta de suma importancia para utilizar sus potenciales combinados para describir y explicar los fenómenos que interactúan y se relacionan con el riesgo.

La materialización de los fenómenos observados ha tenido el espacio como punto de partida desde los primeros recorridos del pensamiento científico. René Descartes ligó el espacio con la geometría, como el que señala la situación en forma más expresa que el tamaño o figura. Posteriormente, Newton concluyó que el espacio era propiedad de una sustancia y, en contraposición, Leibniz sugirió que era algo relativo, un orden de coexistencias, ya que el tiempo es un orden de sucesiones. Kant refuta esa aseveración, al plantear al espacio como cósmico y absoluto; y especificó que el orden de sucesiones podía ser explicado como regiones. Esta distinción llevó, posteriormente, a definir subconceptos heurísticos del espacio, tal y como se conoce el de región o lugar (que tiene una asociación más directa con lo señalado por Descartes) y territorio (Martínez, 2011).

La propuesta de Peter Weichhart y Karl-Michael Höferl (2013) afirma que es necesario concebir al espacio desde una perspectiva científica, como una manera particular de entender el mundo real, cualquiera que sea ésta, y no como una entidad que puede distinguirse de otra o que puede conducir a especulaciones metafísicas. Como resultado de estos planteamientos, se determinan seis tipos de espacios, que se describirán a continuación.

1) El espacio región, es aquel donde se designan áreas de la superficie terrestre que se caracterizan por atributos dominantes específicos; se utiliza para indicar una parte específica de la superficie terrestre cuyos límites no están precisamente definidos y permanecen más o menos borrosos o que se especifican de manera convencional o pragmática. Por ejemplo, regiones de montaña, regiones de aglomeraciones, o regiones periféricas

2) El espacio contenedor, refiere al espacio como un objeto, como un vacío, una estructura. De acuerdo con Newton, este concepto debe considerarse como una especie de contenedor tridimensional en el que todas las cosas materiales están incrustadas. En este tipo de espacio, es donde se construyen las leyes espaciales.

3) El espacio lógico, es aquel que representa las relaciones inmateriales. Se refiere a una estructura sistémica racional que permite la localización de los elementos definidos de un sistema de pensamiento. Estos espacios son producto de prácticas que comprenden tanto modos de pensar como modos de actuar. Analizar estas prácticas consiste en desentrañar las relaciones de poder que las hacen posibles. Por ejemplo: los espacios de color, entre otros.

4) Espacio relativista. Se define como la relación entre las cosas materiales y los cuerpos. Es un concepto relativista porque no requiere la idea de "un espacio vacio o como contenedor" o como un "objeto", éste se basa en la noción de que el espacio se constituye exclusivamente por las relaciones entre las cosas materiales y se desarrolla por la relación posicional de los elementos del mundo material. Por ejemplo, a lado, cerca, lejos, encima, debajo, izquierda o derecha. Este tipo de espacio no se concibe como una estructura autónoma, sino como un atributo de los elementos materiales y los cuerpos, se habla de una espacialidad y no de espacio. Por ello, cada vez que se trata con aspectos materiales de los fenómenos sociales, se puede utilizar este concepto.

A raíz de lo anterior y como resultado de la interacción entre espacio y riesgo, se habla de una espacialidad del riesgo y no de un espacio, para referirse a los aspectos materiales de los fenómenos sociales y la diversidad de manifestaciones territoriales del riesgo consecuentes.

5) El espacio percibido. Refiere a la percepción subjetiva y específica del grupo y la cognición del primer espacio, el objetivo. Es el espacio experimentado, que se encuentra directamente vinculado con un espacio representado $u$ objetivado por el sujeto que lo piensa, pero en términos de contenido, incluye mucho más que una dirección específica del sitio, ya que en éste se desarrollan significados subjetivos. Este tipo de espacio es visto como un concepto cognitivo que significa una interpretación emocional específica de alguna parte espacialmente definida del mundo vital subjetivo o colectivo. 
Para la Geografía Humana, este espacio se denomina lugar.

6) El espacio vivido, es el producto de una construcción social, es una entidad muy compleja que emerge a través de la práctica de la atribución social, la apropiación y la producción. Kant interpretó al espacio como una especie de precondición necesaria para la posibilidad de concebir al mundo. Este último espacio, es un resultado del proceso de regionalización cotidiana; la práctica social y económica produce configuraciones específicas de las cosas dentro del mundo material. $Y$ también es una derivación de la interacción de los conceptos de espacio objetivo y al espacio contenedor, principalmente. Aunque, no en todos los casos, también se refiere al espacio relativista. Tales espacios son producidos y construidos por procesos cognitivos y por las acciones de actores individuales o colectivos.

Desde esta misma visión pragmática del lenguaje, mientras el espacio se concibe como un concepto categórico, el término de riesgo es considerado como un término relacional, ya que cuando se habla de riesgo, es necesario incluir múltiples relaciones con otras entidades - conceptos que son indispensables Para el caso específico de esta reflexión, el riesgo es el vínculo que relaciona a la espacialidad cultural con el territorio.

Se pueden distinguir cuatro diferentes construcciones categoriales válidas y viables para definir y medir el riesgo, y cada una de ellas se puede relacionar con los diversos conceptos de espacio antes mencionados, de los cuales todos son útiles para la exploración de riesgos: -. El espacio objetivo es necesario para describir la distribución de la ocurrencia de un evento que cause daños y sus consecuencias.

-. El espacio contenedor, es indispensable para hacer declaraciones sobre la cantidad de daño por la materialización del riesgo, causando un evento o desastre específico.

-. El espacio lógico, es útil al clasificar riesgos sistémicos. -. Los espacios percibidos y vividos, para las investigaciones sobre riesgos, se le debe atribuir una importancia particular, ya que para referirse a eventos dañinos es necesario comprender la construcción social del territorio y los aspectos materiales e inmateriales de áreas seguras e inseguras, entre otros aspectos de orden territorial.

Así, los diferentes acercamientos ofrecen distintas perspectivas epistémicas de los fenómenos que trastocan la manera en cómo se revisan los espacios con relación al riesgo. No existe una forma única de representar o abordar los fenómenos asociados a los riesgos, amenazas, vulnerabilidad o desastres. Es por ello que en esta propuesta, al utilizar esta pluralidad de conceptos y acercamientos espaciales, como perspectivas epistémicas integrales y relacionadas entre sí, es posible generar nuevas contribuciones geográficas que inciten al debate sobre la investigación de riesgos. Además, ofrece la oportunidad de examinar diferentes dimensiones de estos fenómenos, a través de los objetos particulares de estudio; desde varias disciplinas y ciencias para entonces, al final, poder obtener una visión integral y holística de este fenómeno que modifica territorios y dinámicas económicas, y socio culturales dando lugar a una producción socioespacial específica de cada lugar (Figura 1).

Como se puede identificar, en la figura 1, el territorio, siempre como una construcción social, es la base para la comprensión de la estructuración actual de la formación socioespacial. Es decir, toda relación social que incide en el territorio y se expresa como territorialidad, donde interactúan las relaciones sociales, los espacios de poder, de gestión y de dominio del estado, de los individuos de grupos y organizaciones.

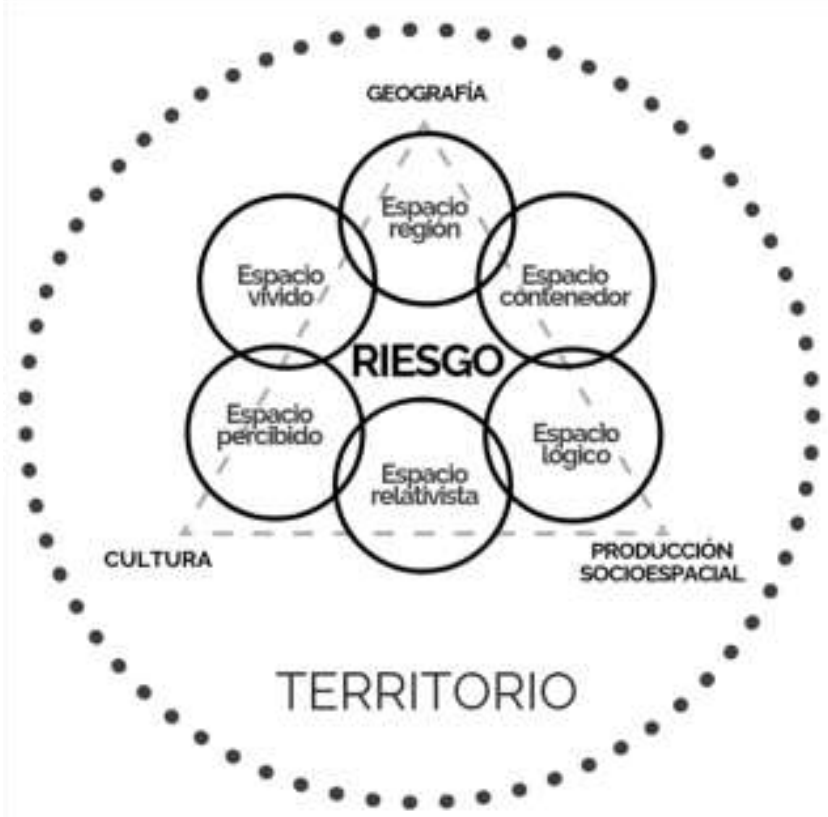

Figura 1. Propuesta para analizar a la espacialidad cultural del riesgo

Fuente: Elaboración propia adaptada de Weichhart, Peter y Höferl, Karl-Michael en Müller-Mahn (ed.) (2013), The Spatial Dimension of Risk How geography shapes the emergence of riskscapes. Routledge, New York.

La dimensión espacial es esencial para la construcción social del riesgo; los aspectos mencionados, pueden ser un elemento más a considerarse para la toma de decisiones de la sociedad, el ordenamiento territorial, la 
gestión del riesgo y ambiental, para una planificación del espacio. Analizar el riesgo y el espacio, en conjunto, es un desafío para el cual la Geografía está bien equipada a través de su perspectiva integrada en el espacio físico y social (Müller-Mahn, Everts y Doevenspeck, 2013). Sin embargo, esta propuesta queda abierta para continuar profundizando en ella y para ponerse en práctica.

\section{Consideraciones finales}

El riesgo se ha convertido en un concepto cultural y político central, en el que se organizan individuos, grupos sociales e instituciones. Las condiciones de vulnerabilidad existen y están relacionadas con el tiempo y el espacio, como resultado de los factores socioculturales. Cuando hablamos de espacialidad cultural se piensa en favorecer la percepción y representación del riesgo diseñando planes de acciones culturales que impacten a la sociedad (comunidad).

La propuesta es el resultado de este documento; desde una visión territorial, se reconoce que el riesgo y el espacio no existen independientemente de la percepción y la acción humana. Sin embargo, tanto el riesgo como el espacio se caracterizan por componentes objetivos y subjetivos, y ambos se refieren a mundos tanto materiales como imaginarios. En consecuencia, un análisis integrado requiere ambos conceptos físicos y sociales. Las perspectivas integrales y espaciales del riesgo también se complementan cuando se trata de su aplicación práctica, por ejemplo en la gestión de riesgos. Aunado a lo anterior, otra de las fortalezas de la Geografía es que puede construir y deconstruir imágenes cartográficas al mismo tiempo. Mientras que la cartografía puede ser utilizada como una herramienta importante para representar la variación geográfica del riesgo y sus alcances, sus productos también son un valioso instrumento de interrogación crítica, ya que no sólo representan sino que también crean espacio.

La comprensión del espacio y sus implicaciones prácticas han cambiado en la transición de las estrategias tradicionales de gestión de riesgos a un concepto más amplio y más completo, al incluir las diversas interpretaciones del espacio. Es importante considerar las múltiples formas en que diferentes grupos sociales, partes interesadas y expertos, relacionan sus evaluaciones subjetivas del riesgo con un marco espacial. En cierto modo, las dimensiones espaciales del riesgo son tan diversas como la cantidad de puntos de vista diferentes sobre el riesgo y el espacio o los paisajes y territorios del riesgo. Cualquier investigación relevante, significativa y pertinente, la práctica de la comunicación y la gestión debe tener en cuenta la diversidad y la multiplicidad de riesgos, donde el espacio modela los riesgos y éstos al mismo tiempo dan forma al espacio.
Mediante el ejercicio anterior, es posible observar cómo el espacio físico y el espacio social se superponen de maneras significativas. Las dimensiones sociales y espaciales del riesgo se desarrollan de forma diferente en diversos lugares. Las formas en que se comprenden, identifican y tratan los riesgos se relacionan con el lugar específico y las implicaciones importantes para la comprensión del riesgo. No se debe olvidar que lo real y lo imaginario se basa en la experiencia individual y colectiva, la tradición y el conocimiento. Reflejan normas culturales, preferencias y significado simbólico, pero también representaciones contradictorias de límites y territorios disputados. Las representaciones contradictorias del espacio son una importante fuente de incertidumbre en estos paisajes de riesgo.

Tanto los espacios físicos como los sociales de la producción de riesgos son de relevancia práctica. Cualquier acción dirigida a reducir el riesgo debe tener en cuenta las causas y los mecanismos de los procesos en el mundo material, así como las percepciones, las dinámicas socioculturales y la manera en que las sociedades reaccionan frente a amenazas potenciales. Cada riesgo se desarrolla de manera diferente, produciendo desigualdades sociales y espaciales de acuerdo con su contexto y realidad local.

Esta propuesta incide no como un único método, sino como una opción más dentro de una multitud de enfoques metodológicos y herramientas para estudiar la espacialidad del riesgo, las asignaciones, estadística, probabilidad y cuestionarios han sido parte del conjunto de herramientas geográficas durante mucho tiempo. Más recientemente, las metodologías cualitativas han despertado más interés, por ejemplo entrevistas abiertas - semiestructuradas, observación participante, cartografía de la percepción y escritura etnográfica entre otros.

Sin duda, es necesario establecer una visión integral, que consistirá en un análisis crítico que identifique y explique aquellas categorías de estudio, mediante estudios de caso, lo que aportará también un modelo teórico-conceptual y una propuesta metodológica verificada y evaluada, que proporcione horizontes novedosos a la investigación sobre la espacialidad cultural del riesgo y para otras que se realicen sobre la temática de la gestión del riesgo.

\section{Referencias Bibliográficas}

Aneas de Castro, Susana (2000). Riesgos y peligros, una visión desde la Geografía, Scripta Nova. Revista Electrónica de Geografía y Ciencias Sociales. No. 60. Universidad de Barcelona.

García-Tornel, Francisco (1984). La geografía de los riesgos. Geocrítica, Año IX, Número 54, noviembre de 1984. 
Calvo García-Tornel, Francisco (2000). Panorama de los estudios sobre riesgos naturales en la geografía española, Boletín de la A.G.E. no. 30, pp. 21-35.

Capel, Horacio (2010). Francisco Calvo García-Tornel: Los riesgos de la geografía y la geografía de los riesgos. Papeles de Geografía, Sin mes, pp. 11-22.

Cardona, Omar Darío. (2001). Estimación Holística del Riesgo Sísmico Utilizando Sistemas Dinámicos Complejos. Tesis Doctoral, Universitat Politècnica de Catalunya.

Castells, Manuel (2001). La galaxia Internet. Barcelona. Areté Plaza\&Janés.

Garrocho Rangel, Carlos, Buzai, Gustavo (Coords.) (2015). Geografía aplicada en Iberoamérica. Avances, retos y perspectivas. El Colegio Mexiquense. México.

Hardy Casado, Virginia; Cuevas Muñiz Alicia; Gallardo Milanés, Olga (2019). Aprendizaje y resiliencia en la gestión local de riesgos de desastres. Luz, v. 18, n. 2, pp. 42-52.

Hewitt, Kenneth. (1997). Regions at Risk. A Geographical Introduction to disasters. Harlow: Longman.

Krüger Fred., Bankoff Greg, Cannon Terry, Orlowski Benedikt y Schipper Lisa (eds.) (2015). Cultures and disasters. Understanding cultural framings in disaster risk reduction, New York, Routledge.

Martínez Apráez, Laura (2019). Víctimas, salvadores y el colapso de una ONG: beneficios y riesgos de la aplicación de metodologías participativas en el trabajo de organizaciones no gubernamentales. Antípoda. Revista de Antropología y Arqueología, n.o 35, pp. 47-67.

Martínez Rubiano, Martha (2011). Los geógrafos y la teoría de riesgos y desastres ambientales. Perspectiva Geográfica, no. 14, pp. 241263.

Müller-Mahn, Detlef (ed.) (2013). The Spatial Dimension of Risk. How geography shapes the emergence of riskscapes. Routledge, New York.

Ortega Valcárcel, José. (2000). Los horizontes de la Geografía: Teoría de la Geografía. Ariel Geografía, Barcelona.

Rojas Vilches, Octavio; Martínez Reyes, Carolina (2011). Riesgos naturales: evolución y modelos conceptuales. Revista Universitaria de Geografía, Bahía Blanca, v. 20, no. 1, 2011.

Toscana Aparicio, Alejandra. (2006). La incorporación y representaciones espaciales del Nuevo Mundo en el Viejo Mundo. Investigaciones Geográficas, Boletín del Instituto de Geografía, UNAM, no. 59, pp. 113-122.

Toscana Aparicio, Alejandra, Valdez Pérez, Verónica (2014). Representaciones sociales del desastre de 1940 en Santa Cruz Pueblo Nuevo, Estado de México. Investigaciones Geográficas, Boletín del Instituto de Geografía, no. 83, 2014, pp. 88-101.

United Nations Office for Disaster Risk Reduction (UNISDR) (2015). Marco de Sendai para la Reducción del Riesgo de Desastres 2015-2030.

Vargas Ulate, Gilbert (2012). Espacio y Territorio en el análisis Geográfico, Reflexiones, vol. 91, núm. 1, 2012, pp. 313-326 Universidad de Costa Rica. 